\title{
Increase in the secretion of pro-inflammatory cytokines by peritoneal leucocytes in a genetic mouse model of obesity and metabolic syndrome
}

\author{
N. M. de Castro, J. Cruces and M. De La Fuente \\ Department of Physiology, Faculty of Biology, Complutense University of Madrid, C/José Antonio Novais, 2, 28040 \\ Madrid, Spain
}

Metabolic syndrome is characterised by a low-grade chronic inflammation involving white adipose tissue ${ }^{(1)}$. Db/db mice show a mutation in the leptin receptor gene and are a model of genetic obesity as well as metabolic syndrome ${ }^{(2)}$. Previous studies have shown an impairment of the lymphoproliferative response in these obese mice at two months of age, which seems to show a premature immunosenescence. It is well known that with ageing there is an imbalance in the secretion of cytokines ${ }^{(3,4)}$ with higher levels of proinflammatory (PI) (such as TNF-alpha and IL-1beta) compared to the anti-inflammatory (AI) (for example, IL-10) cytokines. For this reason, we have investigated the secretion of these cytokines by leucocytes in young obese mice. Female genetically obese mice (db/db), lean heterocygotic mice $(\mathrm{db} /+)$ and wild type mice $(+/+)$ fed with a standard diet were used. The peritoneal leucocytes of these animals at two months of age were obtained and maintained in a culture supplied with lipopolysaccharide (LPS) of E.coli. After 24 hours the culture medium was collected and the secretions of IL-10, IL-1beta and TNF-alpha were measured by luminometry. The results showed that the secretions of IL-10 were similar in the three groups studied. However, the secretions of TNF-alpha and IL-1beta were higher $(p<0.05$ and $p<0.01$, respectively) in obese mice compared to wild control animals. In conclusion, young obese mice showed high levels of PI cytokines and this early PI/AI imbalance might contribute to the chronic inflammatory status associated with obesity and to the development of premature oxi-inflamm-ageing in these animals.

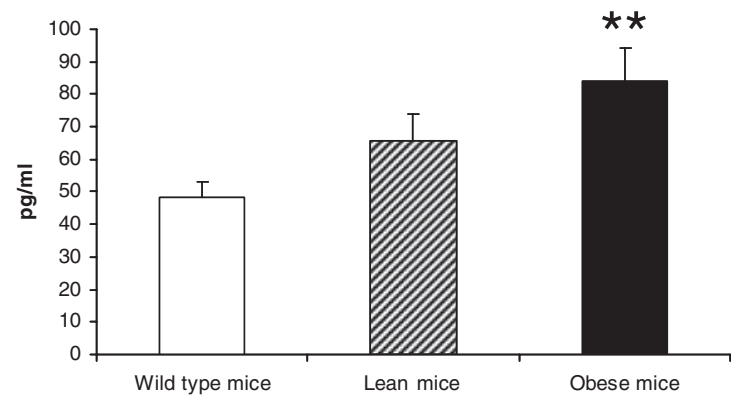

Figure 1. IL-1 BETA.

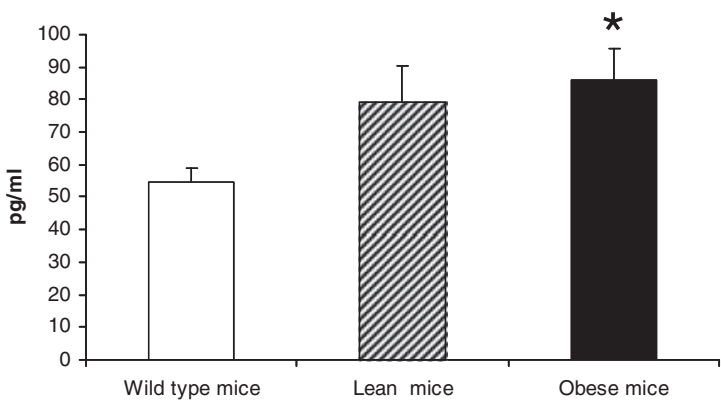

Figure 2. TNF-ALPHA

Data are expressed as mean $\pm \mathrm{SE} * p<0.05 ; * * p<0.01$ with respect to the Wild type group.

Financial support: MICINN (BFU2011-30336), UCM Research Group (910379ENEROINN) and RETICEF. (RD06/0013/0003) (ISCIII) of Spain.

1. Wisse BE. The inflammatory syndrome: the role of adipose tissue cytokines in metabolic disorders linked to obesity. J Am Soc Nephrol. 2004 Nov; 15 (11): 2792-800.

2. Palmer G, Aurrand-Lions M, Contassot E. et al. Indirect effects of leptin receptor deficiency on lymphocyte populations and immune response in db/db mice. J Immunol. 2006 Sep 1; 177 (5): 2899-907.

3. Bruunsgaard H, Pedersen M, Pedersen BK. Aging and proinflammatory cytokines. Curr Opin Hematol. 2001 May; 8 (3): 131-6.

4. Arranz L, Lord JM, De la Fuente M. Preserved ex vivo inflammatory status and cytokine responses in naturally long-lived mice. Age (Dordr). 2010 Dec; 32 (4): 451-66. 